\title{
Cultura Física como proyección de salud, en estudiantes de ECTAFIDE
}

\section{Physical culture as a health projection, in ECTAFIDE students}

Recibido 25/09/2020

Gabriela Inés Jiménez Enriquez

Universidad de San Carlos de Guatemala

gabinesjimenez9979@gmail.com

https://orcid.org/0000-0001-6198-7089

Aceptado 18/01/2021

\section{Referencia}

Jiménez Enriquez, G. I. (2021). Cultura Física como proyección de salud, en estudiantes de ECTAFIDE. Revista Docencia Universitaria, 2(1), 40-50. https://doi.org/10.46954/revistadusac.v2i1.23

\section{Resumen}

Los estudiantes de la Escuela de Ciencia y Tecnología de la Actividad Física y el Deporte (ECTAFIDE), de la Universidad de San Carlos de Guatemala, estudiaron para adquirir conocimientos teóricos-prácticos que los ayuden a desarrollar habilidades y capacidades físico-deportivas en el ser humano, fomentando la Cultura Física en la sociedad. Fortalecieron las habilidades y aptitudes específicas de la carrera porque utilizan el cuerpo para transmitir, explicar, demostrar y corregir las habilidadescapacidades, en sus diferentes modalidades.

El objetivo fue describir cómo los estudiantes adquirieron y conservaron la cultura física como proyección de salud, siendo los objetivos específicos, identificar qué actividades realizaron los estudiantes para alcanzar el hábito de la actividad física y establecer cómo conservaron la cultura física. Se utilizó el enfoque cuantitativo, descriptivo y se aplicó la encuesta en una muestra de 100 estudiantes de los tres primeros años. Los resultados demostraron que el $93 \%$ de los estudiados realizan 
actividad física, el $80 \%$ realiza actividad física tres veces a la semana y diariamente y el $100 \%$ practica actividad física por salud. Concluyendo que los estudiantes, conservan la cultura física y proyectan salud, aplicando lo aprendido en su diario vivir.

\section{Abstract}

The students of the School of Science and Technology of Physical Activity and Sports (ECTAFIDE), of the University of San Carlos de Guatemala, studied to acquire theoretical-practical knowledge that will help them develop skills and physical-sports capacities in the being human, promoting physical culture in society. They strengthened the specific skills and aptitudes of the career because they use the body to transmit, explain, demonstrate and correct the skills-abilities, in their different modalities.

The objective was to describe how the students acquired and preserved physical culture as a health projection, the specific objectives being to identify what activities the students did to achieve the habit of physical activity and establish how they preserved physical culture. The quantitative, descriptive approach was used and the survey was applied to a sample of 100 students from the first three years. The results showed that $93 \%$ of those studied perform physical activity, $80 \%$ perform physical activity three times a week and daily and $100 \%$ practice physical activity for their health. Concluding that the students preserve the physical culture and project health, applying what they have learned in their daily lives.

\section{Introducción}

Los estudiantes de la Escuela de Ciencia y Tecnología de la Actividad Física y el Deporte (ECTAFIDE), se profesionalizan en la aplicación de metodologías para la actividad física, que optimicen la salud física y mental del ser humano mejorando la calidad de vida. Los futuros profesionales, deben mantener el hábito de la actividad física, siendo activos constantemente, cuentan con la destreza motriz para transmitir los conocimientos y habilidades hacia sus practicantes y se completa con la proyección de salud y calidad de vida, basada en la práctica dinámica de hábitos de ejercicio que le permitan desarrollarse óptimamente en su vida cotidiana.
Palabras clave:

actividad física, educación física, cultura física, salud.

Keywords:

physical activity, physical education, physical culture, health. 
Alcanzar el hábito de actividad física, requiere de constancia, dedicación y disciplina, para consolidar la cultura física como parte fundamental de su cultura general, iniciándose por medio de la clase de $E F$, ya que el aprendizaje de esta especialización, se centra en la formación de hábitos, siendo el principal, el hábito del ejercicio físico, que garantice que después de su proceso de aprendizaje de 12 o 13 años de formación, estos hábitos de ejercitación sean perdurables y garanticen una calidad de vida. Se utilizó el enfoque cuantitativo, descriptivo, aplicando la encuesta a una muestra de 100 estudiantes de los tres primeros años de ECTAFIDE.

\section{Justificación}

Los estudiantes, por la riqueza teórica-práctica de la carrera, deben poseer cualidades, habilidades y aptitudes específicas, que le permitan transmitir, explicar, demostrar y corregir las habilidades motrices y técnico-deportivas a sus practicantes. En la actualidad, se está perdiendo el interés por la práctica de la actividad física, por las condiciones socioculturales, económicas y académicas que se presentan en los contextos de la sociedad, del mismo modo por las exigencias que la misma impone, con un ritmo de vida acelerada y tecnológicamente activa, que limita el tiempo de ocio y convivencia social.

Los estudiantes de ECTAFIDE al ser líderes, contribuyen en la sociedad, creando conciencia y sensibilizando sobre la actividad física al "practicar lo que predican", de una forma sistemática y periódica en su rutina diaria, demostrando que poseen cultura física, objetivo que todo educador del movimiento quiere obtener para sí mismo, estudiantes, amigos, familiares y la comunidad.

El objetivo general de la investigación, es describir cómo los estudiantes de ECTAFIDE adquieren y conservan la cultura física como proyección de salud, los objetivos específicos, identificar qué actividades realizan para alcanzar el hábito de la actividad física y establecer cómo conservan la cultura física. Se realizó esta investigación, para sensibilizar y concientizar a los estudiantes, sobre cultura física e iniciar el desarrollo de investigación en este campo de estudio que pueda extrapolarse a otras áreas de actividad profesional. 


\section{Materiales y métodos}

Se utilizó el enfoque cuantitativo, descriptivo, a través de un diseño no experimental transeccional descriptivo, se aplicó la encuesta a estudiantes de los tres primeros años de ECTAFIDE, utilizando una muestra de 100 estudiantes, de una población de 135.

\section{Resultados}

Figura 1. ¿Realiza actividad física?

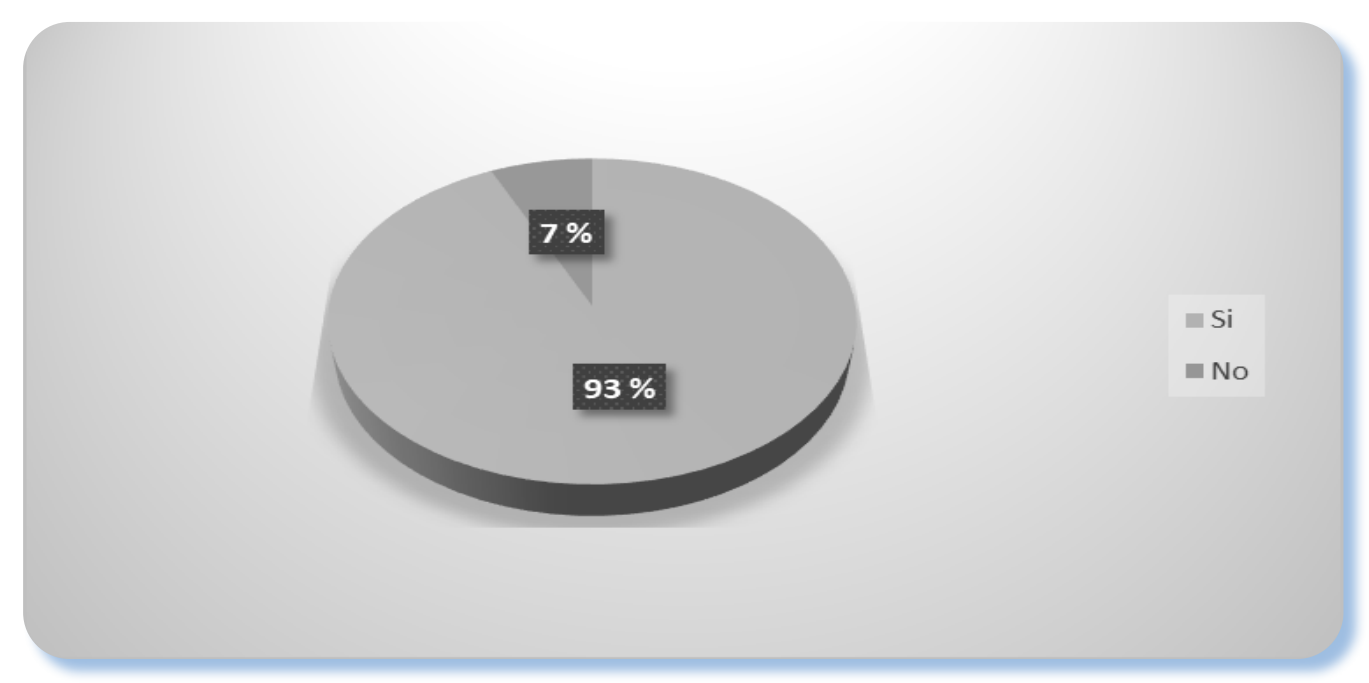

Fuente: elaboración propia de encuesta aplicada a estudiantes de ECTAFIDE, octubre de 2018.

La figura uno, refleja que el $93 \%$ de los estudiantes, si realizan Actividad Física dentro de su rutina diaria, siendo alentador y positivo, ya que, a pesar de ser estudiantes universitarios con responsabilidades, eligen un tiempo adecuado para realizar actividad física, demostrando que están conscientes de los múltiples beneficios que brinda la actividad física, por consiguiente, este hábito está fortalecido. Por otro lado, únicamente el siete \% de los estudiantes, no realizan ninguna actividad física dentro de su rutina diaria, especificando que los motivos de su inactividad es la pereza y la falta de tiempo. 


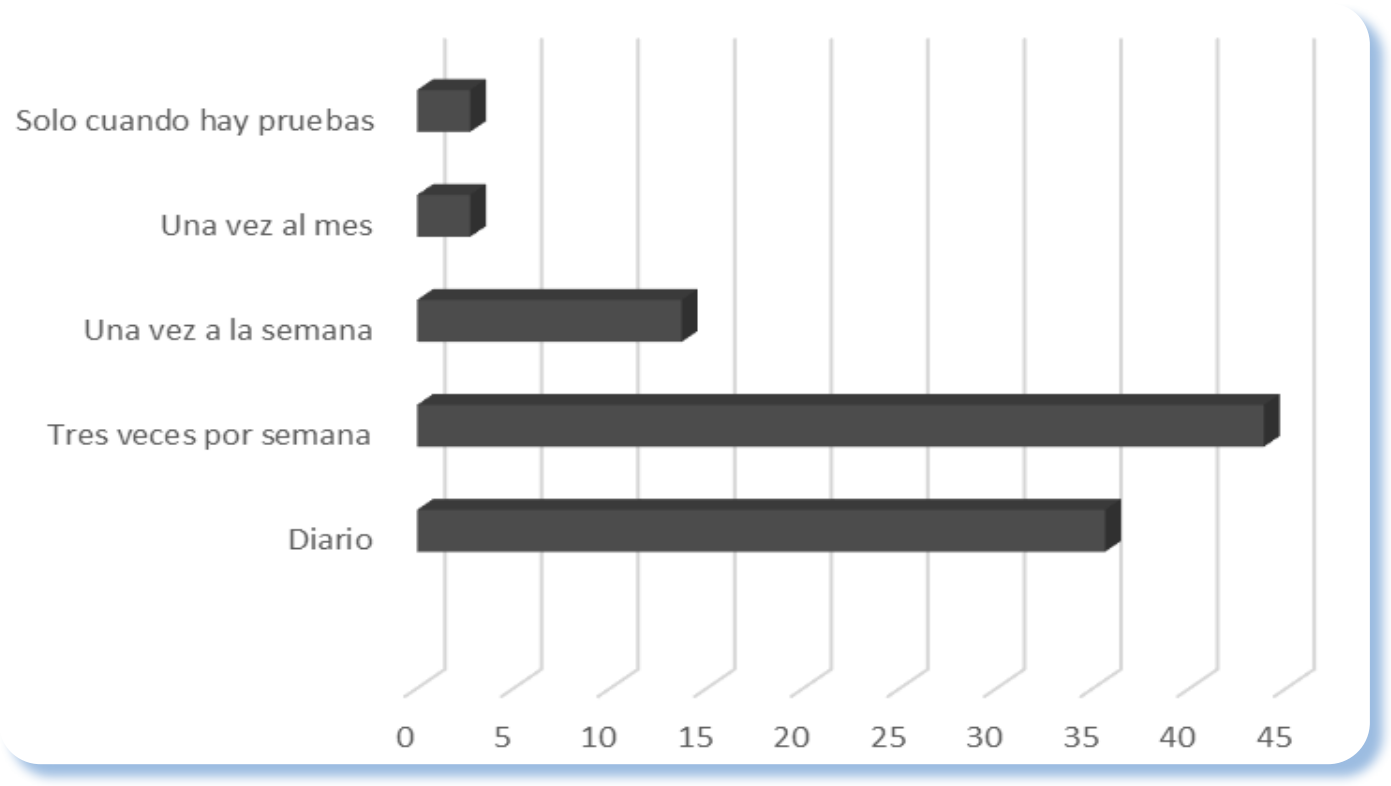

Fuente: elaboración propia de encuesta aplicada a estudiantes de ECTAFIDE, octubre de 2018.

La figura dos, especifica que el $44 \%$ de los estudiantes, practican actividad física tres días a la semana, 37 \% la practican diariamente. Confirmando la importancia que le dan a la práctica constante de actividad física dentro de su rutina, sumando $81 \%$ los estudiantes que mantienen la cultura física con una periodización aceptable, como lo que recomienda la OMS.

Otros datos, demuestran que el $14 \%$ de los estudiantes, realizan actividad física tan solo una vez a la semana, el tres \% practica una vez al mes, el tres \% únicamente realiza actividad física cuando hay pruebas, cumpliendo el precepto del colegio Americano de Medicina del Deporte de "hacer algo de actividad física es mejor que no hacer nada". Estos resultados engloban el $21 \%$ de estudiantes que no han generado el hábito de la actividad física dentro de su rutina diaria, aspecto que debe mejorarse, proporcionando información, promoviendo actividades y motivando a los estudiantes a cambiar su estilo de vida. 
Figura 3. Orden de preferencia de la actividad física

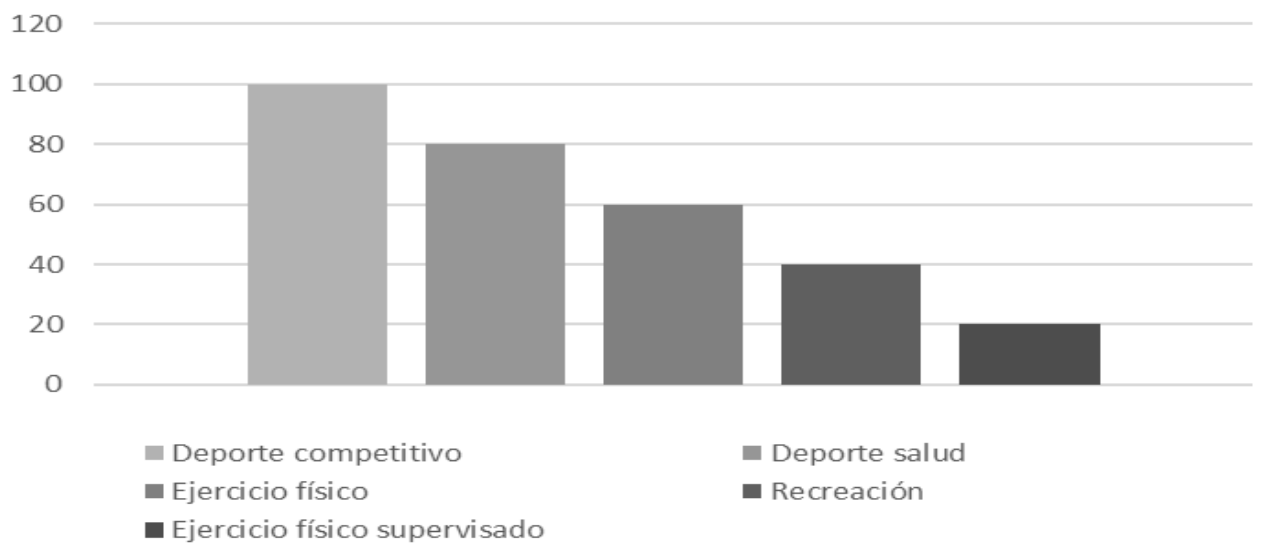

Fuente: elaboración propia de encuesta aplicada a estudiantes de ECTAFIDE, octubre de 2018.

En la figura tres, se preguntó a los estudiantes que seleccionaran en orden de preferencia las actividades físicas, siendo la favorita de los estudiantes el deporte competitivo (deporte federado), entendiéndose como la participación periódica en entrenamientos y competiciones sistematizadas en las asociaciones y federaciones deportivas, donde el atleta debe mejorar su condición física como su habilidad técnica deportiva, para obtener resultados satisfactorios en las competencias programadas dentro del macrociclo de entrenamiento, entrenan para aprender, entrenan para competir y entrenan para ganar, como lo plasma los objetivos en las etapas tres, cuatro, cinco y seis del Plan nacional del deporte, educación física y recreación física 20142024. Consejo Nacional del Deporte Educación Física y la Recreación. [CONADER] (2016).

En segundo lugar, el deporte salud (deporte no federado), participando en torneos o campeonatos locales, municipales, universitarios y lúdicos, en compañía de amigos y familiares, ocupando su tiempo libre con la finalidad de mantenerse activo y saludable, cumpliendo con la etapa dos del Plan nacional del deporte, educación física y recreación física 2014-2024, con el objetivo de competir para recrearse y consolidar gestos motrices CONADER (2016). 
En tercer lugar, el ejercicio físico, mejorando considerablemente la condición física, o capacidades condicionantes por su intencionalidad y sistematización y también de acuerdo a la individualidad, finalidad e interés del ejecutante. Siendo el principal hábito, en el currículo de Educación física, que se pretende desarrollar a través de un proceso de enseñanza-aprendizaje. En cuarto lugar, la recreación, siendo la quinta tarea de la $\mathrm{EF}$, en la misión pedagógica de la misma y por último el ejercicio físico supervisado, o sea, la práctica de la actividad física supervisada por un entrenador personal asistiendo a un gimnasio particular, con el objetivo de satisfacer determinadas exigencias de rendimiento físico.

\section{Discusión}

Los resultados indican que los estudiantes de ECTAFIDE, si practican la Actividad Física, en un 93 \% (hombres y mujeres), en el estudio presentado por Pérez Ugidos et al. (2014) manifiesta que el $75.3 \%$ de los estudiantes de Educación Física, realizan altos niveles de Actividad física, en comparación con otras carreras universitarias, Farinola (2012) menciona que el $87 \%$ de los estudiantes tuvieron un alto nivel de actividad física, por lo tanto, los estudiantes de esta especialización realizan actividad física periódicamente.

Los estudiantes ejecutan la actividad física por la mañana en un 44 $\%$, al aprovechar el mejor momento del día para la ejercitación, $25 \%$ ejecuta la actividad física por la tarde, $11 \%$ por la noche y $12 \%$ utiliza un horario flexible, consolidando el compromiso por desarrollar y mejorar sus capacidades físicas y técnicas deportivas. Se encuentran diferencias con el estudio de Pavón (como se citó en Hellín Gómez 2003), ya que por la falta de tiempo libre los estudiantes ocupan el horario de 19:00 a 20:00 horas para realizar actividad física.

Dentro de las actividades físicas que prefieren los estudiantes se identificaron: Deporte competitivo, (deporte federado), Deporte salud (deporte no federado) Ejercicio físico, Recreación y el Ejercicio supervisado, en el estudio presentado por Pérez et al. (2005) se manifiesta que los estudiantes de la Facultad de Ciencias de la Actividad física de la Universidad De León, también prefieren el deporte especialmente el fútbol y fútbol sala. 
La OMS (2020) recomienda que los adultos entre los 18 a 64 años de edad, realicen actividades físicas aeróbicas moderadas durante al menos 150 a 300 minutos o actividades físicas aeróbicas intensas de 75 a 150 minutos durante la semana. Encontrando que los estudiantes conservan la Cultura Física, ya que el cuatro \% realiza 15 minutos, $18 \%$ 30 minutos, $14 \% 45$ minutos, $30 \%$ dedica una hora diaria a la práctica de la actividad física, $21 \%$ dos horas y el 13\% más dos horas; en el estudio de Pérez et al. (2005) se menciona que los estudiantes dedican siete horas a la semana a la práctica de la actividad física, por lo tanto, se encuentran entre el rango de tiempo aceptable que recomienda la OMS.

Es importante recalcar que $46 \%$ de los estudiantes, estructuran correctamente la práctica física: calentamiento, parte principal y vuelta a la calma, demostrando que aplican lo aprendido en la práctica de actividad física, preparando al cuerpo adecuadamente para evitar lesiones y problemas posteriores. También, se preguntó que seleccionaran en orden de prioridad por qué motivo realizan actividad física, demostrando que el $100 \%$ de los estudiantes, priorizan la salud, ya que, reconocen la cultura física, como un hábito importante dentro de su cultura general, al ser conscientes de los beneficios fisiológicos, psicológicos y sociales que genera, como proyección de salud y calidad de vida.

El $80 \%$ de los estudiantes, manifiesta que es su pasatiempo favorito y $60 \%$ lo considera un hábito perenne en su vida. En el estudio de Pavón Lores et al. (2003) se especifica que el motivo principal de los estudiantes para realizar actividad física es la competición, seguidamente la capacidad individual y la aventura, diferencias entre los estudiantes ya que la diversión y la adrenalina es más importante que la salud.

Con base a la Ley de Educación Nacional, la EF tiene finalidades puntuales como preservar y mejorar la salud, adquirir y mantener la aptitud física y deportiva, promover la sana ocupación del tiempo libre y contribuir al desarrollo de los valores morales y al completo bienestar físico, intelectual y social del ser humano. Para tal efecto dispondrá de procesos y medios de planificación, investigación, programación y evaluación propias y específicas. 
Como también, el deporte federado y deporte salud, que son fenómenos socioculturales, ya sea como estilo de vida o espectáculo, la recreación, que brinda gozo y placer al divertirse en el tiempo libre, el ejercicio físico, enfocado en mejorar la condición física por su intencionalidad y sistematización. Cada medio es conocido por los estudiantes de Ectafide, porque se utilizan para estimular la práctica de la actividad física, ofreciendo diversidad de actividades de acuerdo a los intereses, necesidades y capacidades individuales.

Para concluir, el objetivo de la cultura física, es convertirse en parte fundamental de la cultura general de la sociedad y por ende de cada persona, con la intencionalidad de crear conciencia en la práctica de la actividad física, para mejorar su desarrollo físico, la salud y elevar la calidad de vida. Siendo la propuesta, modificar los contenidos en algunos cursos del pensum de estudios, para integrar la cultura física en la formación de los estudiantes de ECTAFIDE, como principal promotora de la actividad física universitaria, creando proyectos de masificación de cultura física orientando a los estudiantes inactivos a través de foros, talleres y festivales, que fortalezcan la cultura física, como pilar fundamental para proyectar salud y calidad de vida.

Está dinámica observada en la formación de profesionales responde al "Plan Nacional del Deporte, Educación Física y Recreación Física 20142024", que propone CONADER, pretende desarrollar estrategias que englobe a todas las entidades de gobierno, en el Proceso sistemático del desarrollo de la cultura física y el deporte, no obstante la puntual estrategia para las universidades y/u otras entidades de formación de profesionales no queda establecida en este plan, dando autonomía de currícula a las federaciones y universidades.

ECTAFIDE, es pieza fundamental, al ser semillero de la cultura física, primero con su ejemplo y luego transformando a los demás, para que Guatemala sea un país activo, saludable y ganador.

\section{Agradecimientos}

A la Universidad de San Carlos de Guatemala, específicamente al coordinador, docentes y estudiantes de la Escuela de Ciencia y Tecnología de la Actividad Física y el Deporte, a mi asesor de tesis Maestro Carlos Humberto Aguilar Mazariegos. Maestra Rogelia Nicté Bravo Navarro y profesor José Raúl Vega por su apoyo incondicional. 


\section{Referencias}

Consejo Nacional del Deporte Educación Física y la Recreación. (2016). Plan Nacional del Deporte, Educación Física y la Recreación 2014-2024.https://conader.com.gt/plan-nacional/

Farinola, Martín Gustavo (2012) Niveles de actividad física en alumnos de la carrera de profesorado universitario en Educación Física de la Universidad de Flores [Tesis de Maestría, Universidad Nacional de Lanús]. http://www.repositoriojmr.unla.edu.ar/ descarga/Tesis/MAMIC/028633_Farinola.df

Hellín Gomez, P. (15 de mayo de 2003) Hábitos físico-deportivos en la Región de Murcia: implicaciones para la elaboración del currículum en el ciclo formativo de actividades físicodeportivas. [Tesis Doctoral, Universidad de Murcia] Repositorio Institucional de la Universidad de Murcia. http:// hdl.handle.net/10201/373

Organización Mundial de la Salud (26 de noviembre de 2020) Actividad fisica. https:// www.who.int/es/news-room/factsheets/detail/physical-activity

Pavón Lores, A., et al. (2003). La práctica físico-deportiva en la Universidad. Revista Psicología del Deporte,12(1),39-54. file://C:/Users/Gaby\%20Jim\%C3\%A9nez/ Downloads/ Pavnetal2003.pdf

Pérez, D. et al. (2005) Evolución de Motivaciones, Actitudes y Hábitos de los estudiantes de la Facultad de Ciencias de la Actividad Física y del Deporte de la Universidad de León. Revista Motricidad. European Journal of Human Movement.14,65-79. file://C:/Users/Gaby\%20Jim\%C3\%A9nez/Download/DialnetEvolucionDeMotivacionesActitudesYHabitosDeLosEstud-2279075.pdf

Pérez Ugidos, Guillermo et al. (2014) Actividad física y hábitos de salud en estudiantes universitarios argentinos. Nutrición Hospitalaria, 30(4), 896-904. http://scielo.isciii.es/pdf/nh/ v30n4/26originaldeporteyejercicio01.pdf 


\section{Sobre la autora}

Gabriela Inés Jiménez Enriquez es Licenciada en Educación Física, Deporte y Recreación, estudiante de la carrera de Maestría en Docencia Universitaria, de la Facultad de Humanidades de la Universidad de San Carlos de Guatemala.

\section{Copyright (c) Gabriela Inés Jiménez Enriquez}

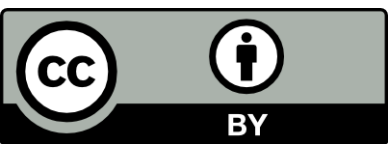

Este texto está protegido por una licencia Creative Commons 4.0.

Usted es libre para compartir, copiar y redistribuir el material en cualquier medio o formato y adaptar el documento, remezclar, transformar y crear a partir del material para cualquier propósito, incluso comercialmente, siempre que cumpla la condición de atribución: usted debe reconocer el crédito de una obra de manera adecuada, proporcionar un enlace a la licencia, e indicar si se han realizado cambios. Puede hacerlo en cualquier forma razonable, pero no de forma tal que sugiera que tiene el apoyo del licenciante o lo recibe por el uso que hace. 